\title{
A COMPUTABLE ABSOLUTELY NORMAL LIOUVILLE NUMBER
}

\author{
VERÓNICA BECHER, PABLO ARIEL HEIBER, AND THEODORE A. SLAMAN
}

Abstract. We give an algorithm that computes an absolutely normal Liouville number.

\section{THE MAIN RESUlT}

The set of Liouville numbers is

$$
\left\{x \in \mathbb{R} \backslash \mathbb{Q}: \forall k \in \mathbb{N}, \exists q \in \mathbb{N}, q>1 \text { and }\|q x\|<q^{-k}\right\}
$$

where $\|x\|=\min \{|x-m|: m \in \mathbb{Z}\}$ is the distance of a real number $x$ to the nearest integer and other notation is as usual. Liouville's constant, $\sum_{k>1} 10^{-k}$, is the standard example of a Liouville number. Though uncountable, the set of Liouville numbers is small, in fact, it is null, both in Lebesgue measure and in Hausdorff dimension (see [6]).

We say that a base is an integer $s$ greater than or equal to 2. A real number $x$ is normal to base $s$ if the sequence $\left(s^{j} x: j \geq 0\right)$ is uniformly distributed in the unit interval modulo one. By Weyl's Criterion [11, $x$ is normal to base $s$ if and only if certain harmonic sums associated with $\left(s^{j} x: j \geq 0\right)$ grow slowly. Absolute normality is normality to every base.

Bugeaud [6] established the existence of absolutely normal Liouville numbers by means of an almost-all argument for an appropriate measure due to Bluhm [3, 4]. The support of this measure is a perfect set, which we call Bluhm's fractal, all of whose irrational elements are Liouville numbers. The Fourier transform of this measure decays quickly enough to ensure that those harmonic sums grow slowly on a set of measure one. Thus, Bugeaud's proof exhibits a nonempty set but does not provide a construction of an absolutely normal Liouville number.

A real number $x$ is computable if there is a base $s$ and an algorithm to output the digits for the base- $s$ expansion of $x$, one after the other. In this note we show the following:

Theorem. There is a computable absolutely normal Liouville number.

Received by the editor January 29, 2014 and, in revised form, April 14, 2014.

2010 Mathematics Subject Classification. Primary 11K16, 68-04; Secondary 11-04.

Key words and phrases. Normal numbers, Liouville numbers, algorithms.

The first and second authors were supported by Agencia Nacional de Promoción Científica y Tecnológica and CONICET, Argentina.

The third author was partially supported by the National Science Foundation, USA, under Grant No. DMS-1001551 and by the Simons Foundation. 
We regard this result as a step into the ancient problem posed by Émile Borel [5] on exhibiting a natural instance of an absolutely normal number. Borel's understanding of "natural" may have been towards numbers that can be described geometrically (as $\pi$ ), analytically (as $e$ ), or algebraically (as $\sqrt{2}$ ). To our mind, algorithmic descriptions are also explicit, immediate and worthy of investigation.

We give an algorithm that determines a real number in the unit interval by recursively constructing a nested sequence of dyadic intervals. At each step the algorithm obtains a new subinterval containing sufficiently many points that satisfy, simultaneously, a better approximation to the Liouville condition, and a better approximation to absolute normality. The number obtained by the algorithm is the unique point in the intersection of these intervals.

The discrepancy of a finite sequence of real numbers is a quantitative indicator of whether its elements are uniformly distributed modulo one in the unit interval. We translate between bounds on harmonic sums and bounds on discrepancy using a discrete version of LeVeque's Inequality (Theorem 2.4 in [9]), proved in Lemma 7.

Like Bugeaud, we use the ingredients of Bluhm's measure. However, we combine those ingredients differently so as to work within subintervals of the unit interval and make explicit the Liouville exponent and the level in Bluhm's fractal. By adapting an argument of Davenport, Erdős and LeVeque [8, we prove that the set of points in a given interval having small harmonic sums has large measure. See Lemma 10.

Our algorithm relies on the fact that the Fourier transform of Bluhm's measure decays not only quickly but also uniformly quickly over all intervals. For any given positive $\epsilon$ there is an extension length $L$ with the following property. Consider any interval the form $\left[p 2^{-a},(p+1) 2^{-a}\right)$, for some nonnegative integer $p$. So, the endpoints have a finite expansion in base 2 , requiring at most $a$ digits. Let $b$ be the counterpart number of digits in the expansion of the left endpoint in base $s$ (precisely, $b=\left\lceil a / \log _{2} s\right\rceil$ ). Then, there is a level in Bluhm's fractal such that for the corresponding measure and for every $\ell$ as large as $L$, the set of reals $x$ in this interval whose harmonic sum associated with $\left(s^{j} x: b \leq j<b+\ell\right)$ is below $\epsilon$, has large measure. We prove this in Lemma 11.

In addition, we exploit another feature of discrepancy: as a function of finite sequences $\left(s^{j} x: a \leq j<b\right)$, it is continuous in two ways. One is with respect to the real variable $x$. That is, for any real numbers such that $|x-y|$ is small, if $\left(s^{j} x: a \leq j<b\right)$ has small discrepancy then $\left(s^{j} y: a \leq j<b\right)$ also has small discrepancy. Lemma 12 formalizes this idea giving quantitative estimates. The second way is with respect to the length of the sequence, given by the variables $a$ and $b$. That is, for any $c$ such that $c-a$ is nonnegative and $c /(b-a)$ is small, if $\left(s^{j} x: a \leq j<b\right)$ has small discrepancy, then both $\left(s^{j} x: a \leq j<b+c\right)$ and $\left(s^{j} x: a-c \leq j<b\right)$ also have small discrepancy. Lemma 13 formalizes this feature in a way that is conveniently applicable.

The algorithm constructs a real number $x$ as the point in the intersection of a nested sequence of dyadic intervals. At each step, the algorithm determines one such dyadic interval, ensuring that the set of real numbers in it has small discrepancy and meets a designated Liouville exponent. However, at each step we do not consider the discrepancy of the entire sequence but the discrepancy of the current extension. Using the mentioned continuity of discrepancy, we conclude that the discrepancy of the limit point $x$ output by the algorithm converges to zero. 
We do not provide bounds on the time complexity for our algorithm. Without the Liouville condition, it is possible to compute absolutely normal numbers efficiently. Specifically, there are algorithms that output the first $n$ digits of an absolutely normal number in time polynomial in $n$. The most efficient of these algorithms requires time just above quadratic [1, where speed is achieved by controlling, at each step, the size of the subinterval and how much progress is done towards absolute normality. The algorithm we present here is not consistent with such a strategy because it does not control the size of the subinterval at each step. So, the estimations of harmonic sums, which are inherently costly, are associated with necessarily long sequences. Constructing Liouville numbers that are normal to a given base, but not necessarily absolutely normal, as done in [10, admits a much simpler approach and can be done in linear time.

\section{Bluhm's measure For COMPuting Liouville numbers}

We write $e(z)$ to denote $e^{z}$. We write the Fourier transform of a real function $f$ as

$$
\widehat{f}(x)=\int_{\mathbb{R}} f(t) e(-2 \pi i x t) d t .
$$

Recall that the Fourier transform of a positive bounded measure $\nu$ is defined, for $x \in \mathbb{R}$, by

$$
\widehat{\nu}(x)=\int_{\mathbb{R}} e(-2 \pi i x t) d \nu(t) .
$$

We write log without subscript for the logarithm in base $e$, and add a subscript for other bases.

2.1. Continuous replacements for step functions. We make use of measures which are supported by subintervals $I$ of $[0,1]$ and have Fourier transforms which decay quickly. Bluhm [3] gives examples of such and we employ them here.

Definition 1. Let $R$ be a real number less than $1 / 2$. Define the function $F_{R}$ on $[-1 / 2,1 / 2]$ by

$$
F_{R}(x)=\frac{15}{16} R^{-5}\left(R^{2}-x^{2}\right)^{2} \text { when }|x| \leq R, \text { and } F_{R}(x)=0 \text { otherwise. }
$$

Let the Fourier series for $F_{R}(x)$ be denoted by

$$
\sum_{n \in \mathbb{Z}} c_{n}^{(R)} e(2 \pi i n x)
$$

Notice that the definition is such that

$$
\int_{\mathbb{R}} F_{R}(x) d x=1 .
$$

As Bluhm points out, the Fourier coefficients

$$
c_{n}^{(R)}=\int_{-1 / 2}^{1 / 2} F_{R}(t) e(-2 \pi i n t) d t
$$

satisfy

$$
c_{0}^{(R)}=1, \quad\left|c_{n}^{(R)}\right| \leq 1, \quad \text { and } \quad\left|c_{n}^{(R)}\right| \leq n^{-2} R^{-2} .
$$


Definition 2. For a subinterval $I$ of $[0,1]$, let $R_{I}$ be such that $4 R_{I}$ is equal to the length of $I$. Let $b$ be the center point of the interval $I$ and let $F_{I}$ be the translation of $F_{R_{I}}$ by $b$, defined as

$$
\begin{aligned}
& F_{I}(x)=\frac{15}{16} R_{I}^{-5}\left(R_{I}{ }^{2}-(x-b)^{2}\right)^{2} \text { when }|x-b| \leq R_{I}, \text { and } \\
& F_{I}(x)=0, \text { otherwise. }
\end{aligned}
$$

The support of $F_{I}$ is contained in $I$ and the analogous inequalities hold for the coefficients of the Fourier series for $F_{I}$,

$$
c_{0}^{(I)}=1, \quad\left|c_{n}^{(I)}\right| \leq 1, \quad \text { and } \quad\left|c_{n}^{(I)}\right| \leq n^{-2} R_{I}^{-2} .
$$

2.2. Bluhm-style measures. Bluhm [3, 4] showed that the set of Liouville numbers supports a Rajchman measure, that is, a positive measure whose Fourier transform vanishes at infinity. Bluhm's measure is the limit of a sequence of measures $\mu_{k}$, for $k \in \mathbb{N}$. The measure $\mu_{k}$ is supported by a set of real numbers $x$ such that there is at least one rational number $p / q$ such that $0<|x-p / q|<1 / q^{k}$. Here, rather than taking a limit of measures, we perform a sequence of finite steps to compute a real $x$ in the limit and argue that for each step there is an appropriate action to take by appealing to an appropriate Bluhm-style measure.

Definition 3 ([3]). For every pair of integers $m$ and $k$ such that $k \geq 1$, let

$$
E(m, k)=\bigcup_{\substack{m \leq q<2 m \\ \text { prime } q}}\left\{x \in \mathbb{R}:\|q x\| \leq q^{-1-k}\right\} .
$$

As usual, we write $C^{2}$ for the class of functions whose first and second derivative both exist and are continuous.

Lemma 4 ([3, Lemma 3.2]). There is a family of $C^{2}$ functions $g_{m, k}$, parametrized by the pairs of positive integers $m$ and $k$, such that the support of $g_{m, k}$ is included in $E(m, k), \widehat{g_{m, k}}(0)=1$, and such that for every function $\Psi$ in $C^{2}$ of compact support, for every positive integer $k$ and for every positive real $\delta$, there is an integer $M=M(\Psi, k, \delta)$ such that for every $m \geq M$ and for every $x \in \mathbb{R}$,

$$
\left|\left(\widehat{\Psi g_{m, k}}\right)(x)-\widehat{\Psi}(x)\right| \leq \delta(1+|x|)^{-1 /(2+k)} \log (e+|x|) \log \log (e+|x|) .
$$

Bluhm defines $g_{m, k}$ by taking the sum of functions $F_{I}$ for appropriate subintervals of those comprising $E(m, k)$ and then normalizing so that $\widehat{g_{m, k}}(0)=1$.

Definition 5. We let $\nu_{I}$ be the measure on $[0,1]$ obtained by integrating $F_{I}$. For $m$ and $k$ positive integers, we let $\nu_{I, m, k}$ be the measure on $[0,1]$ obtained by integrating $F_{I} g_{m, k}$.

Lemma 6. For every subinterval I of $[0,1]$ and every positive integer $k$, there is a positive integer $M$ such that for all $m \geq M, \nu_{I, m, k}(I)=1$.

Proof. By definition of $\nu_{I, m, k}$,

$$
\begin{aligned}
\nu_{I, m, k}(I) & =\int_{I} F_{I} g_{m, k} d t \\
& =\int_{I} F_{I} g_{m, k} e^{-2 \pi i 0 t} d t \\
& =\widehat{F_{I} g_{m, k}}(0) .
\end{aligned}
$$


By Lemma 4, for each positive integer $k$ and each positive real $\delta$, there is an integer $M=M\left(F_{I}, k, \delta\right)$ such that for every $m \geq M$,

$$
\begin{aligned}
\nu_{I, m, k}(I) & \geq \widehat{F_{I}}(0)-\delta(1+|0|)^{-1 /(2+k)} \log (e+|0|) \log \log (e+|0|) \\
& =1-\delta 1^{-1 /(2+k)} \log (e+0) \log \log (e+0) \\
& =1-\delta \log (e) \log (\log (e)) \\
& =1-\delta \log (1) \\
& =1-0 \\
& =1 .
\end{aligned}
$$

Observe that the support of $\nu_{I, m, k}$ is included in the support of $g_{m, k}$, which in turn is included in $E(m, k)$.

\section{LEMMAS}

We say an interval is $s$-adic if it is of the form $\left(p s^{-a},(p+1) s^{-a}\right)$ for nonnegative integers $a$ and $p$. We use $\langle a ; s\rangle$ to denote $\left\lceil a / \log _{2} s\right\rceil$. We write $\{x\}$ to denote the nonintegral part of a real $x$. The cardinality of a set $S$ is denoted by $\# S$.

The discrepancy of a finite sequence $\left(x_{1}, \ldots, x_{n}\right)$ of reals in the unit interval with respect to a fixed interval $[u, v]$ is

$$
D\left([u, v],\left(x_{1}, \ldots, x_{n}\right)\right)=\left|\frac{\#\left\{j: 1 \leq j \leq n \text { and } u \leq x_{j}<v\right\}}{n}-(v-u)\right| .
$$

If we consider its discrepancy with respect to every subinterval in the unit interval, we have

$$
D\left(x_{1}, \ldots, x_{n}\right)=\sup _{0 \leq u<v \leq 1} D\left([u, v],\left(x_{1}, \ldots, x_{n}\right)\right)
$$

Thus, a real number $x$ is normal to base $s$ exactly when the sequence $\left(\left\{s^{j} x\right\}: j \geq 0\right)$ is uniformly distributed in the unit interval, that is,

$$
\lim _{n \rightarrow \infty} D\left(\left\{s^{j} x\right\}: 0 \leq j<n\right)=0 .
$$

Lemma 7 ([2, Lemma 3.8]). For any positive real $\epsilon$ there is a finite set of positive integers $T$ and a positive real $\delta$ such that for any sequence of reals in the unit interval $\left(x_{1}, \ldots, x_{n}\right)$,

$$
\text { if for all } t \in T, \frac{1}{n^{2}}\left|\sum_{j=1}^{n} e\left(t x_{j}\right)\right|^{2}<\delta \text {, then } D\left(x_{1}, \ldots, x_{n}\right)<\epsilon \text {. }
$$

Furthermore, such $T$ and $\delta$ can be computed from $\epsilon$.

Proof. By LeVeque's Inequality (see Theorem 2.4 [9])

$$
D\left(x_{1}, \ldots, x_{n}\right) \leq\left(\frac{6}{\pi^{2}} \sum_{t=1}^{\infty} \frac{1}{t^{2}}\left|\frac{1}{n} \sum_{j=1}^{n} e\left(t x_{j}\right)\right|^{2}\right)^{\frac{1}{3}} .
$$

Since

$$
\left|\frac{1}{n} \sum_{j=1}^{n} e\left(t x_{j}\right)\right|^{2} \leq 1
$$


we get for each positive integer $h$,

$$
\sum_{t=h+1}^{\infty} \frac{1}{t^{2}}\left|\frac{1}{n} \sum_{j=1}^{n} e\left(t x_{j}\right)\right|^{2} \leq \sum_{t=h+1}^{\infty} \frac{1}{t^{2}} \leq \int_{h+1}^{\infty} x^{-2} d x \leq \frac{1}{h+1} .
$$

Assume

$$
\frac{1}{n^{2}}\left|\sum_{j=1}^{n} e\left(t x_{j}\right)\right|^{2}<\frac{\epsilon^{3}}{2}
$$

for all positive integers $t$ less than or equal to $h$. Thus,

$$
\begin{gathered}
\sum_{t=1}^{h} \frac{1}{t^{2}}\left|\frac{1}{n} \sum_{j=1}^{n} e\left(t x_{j}\right)\right|^{2}+\sum_{t=h+1}^{\infty} \frac{1}{t^{2}}\left|\frac{1}{n} \sum_{j=1}^{n} e\left(t x_{j}\right)\right|^{2} \\
\leq \sum_{t=1}^{h} \frac{1}{t^{2}} \frac{\epsilon^{3}}{2}+\frac{1}{h+1} \leq \frac{\epsilon^{3} \pi^{2}}{12}+\frac{1}{h+1} .
\end{gathered}
$$

We can computably choose $h$ so that $\left(6 / \pi^{2}\right)\left(\epsilon^{3} \pi^{2} / 12+1 /(h+1)\right)^{\frac{1}{3}}<\epsilon$. Then, $D\left(x_{1}, \ldots, x_{n}\right)<\epsilon$. This proves the lemma with $T=\{1, \ldots, h\}$ and $\delta=\epsilon^{3} / 2$.

Lemma 8. Let I be a dyadic interval with length $2^{-a}$ and let $k$ be a positive integer. Let $s$ be a base and let $t$ be a positive integer. Then, there is an integer $M$ such that for every $m \geq M$ and every positive integer $\ell$,

$$
\int \frac{1}{\ell^{2}}\left|\sum_{n=\langle a ; s\rangle+1}^{\langle a ; s\rangle+\ell} e\left(2 \pi i t s^{n} x\right)\right|^{2} d \nu_{I, m, k}(x)<\frac{100}{\ell} .
$$

Moreover, $M$ is uniformly computable from $I, k, s$ and $t$.

Proof. Let

$$
Z=\int \frac{1}{\ell^{2}}\left|\sum_{n=\langle a ; s\rangle+1}^{\langle a ; s\rangle+\ell} e\left(2 \pi i t s^{n} y\right)\right|^{2} d \nu_{I, m, k}(y)
$$

Then,

$$
\begin{aligned}
& Z=\int \frac{1}{\ell^{2}} \sum_{n, q=1}^{\ell} e\left(2 \pi i t s^{\langle a ; s\rangle+n} y-2 \pi i t s^{\langle a ; s\rangle+q} y\right) d \nu_{I, m, k}(y) \\
& =\frac{1}{\ell^{2}}\left(\int \sum_{n=1}^{\ell} 1 d \nu_{I, m, k}(y)+\int \sum_{n \neq q} e\left(2 \pi i t s^{\langle a ; s\rangle+n} y-2 \pi i t s^{\langle a ; s\rangle+q} y\right) d \nu_{I, m, k}(y)\right) \\
& =\frac{1}{\ell}+\frac{1}{\ell^{2}} \sum_{n \neq q} \widehat{\nu_{I, m, k}}\left(t s^{\langle a ; s\rangle+n}-t s^{\langle a ; s\rangle+q}\right) .
\end{aligned}
$$

We now give upper bounds for the values of $\widehat{\nu_{I, m, k}}$. Since $R$ is one-fourth of the length of $I$, which is $2^{-a}$, by the above

$$
\widehat{F_{I}}(x) \leq x^{-2} R^{-2}=\frac{2^{2 a+4}}{x^{2}} .
$$

According to Lemma 4, for sufficiently large $m$,

$$
\left|\widehat{g_{m, k} F_{I}}(x)-\widehat{F_{I}}(x)\right| \leq \delta \theta_{k}(x),
$$


where $\delta$ is a constant and can be made arbitrarily small by choice of $m$, and

$$
\theta_{k}(x)=(1+|x|)^{-1 /(2+k)} \log (e+|x|) \log \log (e+|x|) .
$$

Let $c$ be such that

$$
\theta_{k}(x) \leq c(1+|x|)^{-1 / k}
$$

Notice $c$ is uniformly computable from $k$. Thus,

$$
\begin{aligned}
\mid \widehat{\nu_{I, m, k}} & \left(t s^{\langle a ; s\rangle+n}-t s^{\langle a ; s\rangle+q}\right) \mid \\
& =\left|\widehat{g_{m} F_{I}}\left(t s^{\langle a ; s\rangle+n}-t s^{\langle a ; s\rangle+q}\right)\right| \\
& \leq \widehat{F_{I}}\left(t s^{\langle a ; s\rangle+n}-t s^{\langle a ; s\rangle+q}\right)+\delta c\left(1+\left|t s^{\langle a ; s\rangle+n}-t s^{\langle a ; s\rangle+q}\right|\right)^{-1 / k} \\
& \leq \frac{2^{2 a+4}}{\left|t s^{\langle a ; s\rangle+n}-t s^{\langle a ; s\rangle+q}\right|^{2}}+\delta c \frac{1}{\left|t s^{\langle a ; s\rangle+n}-t s^{\langle a ; s\rangle+q}\right|^{1 / k}} . \\
& \leq \frac{2^{4}}{t^{2}\left|s^{n}-s^{q}\right|^{2}}+\frac{\delta c}{t^{1 / k} s^{\langle a ; s\rangle / k}} \frac{1}{\left(s^{n}-s^{q}\right)^{1 / k}} .
\end{aligned}
$$

From which it follows,

$$
Z \leq \frac{1}{\ell}+\frac{2}{\ell^{2}} \sum_{q=1}^{\ell-1} \sum_{n=q+1}^{\ell}\left(\frac{16}{t^{2}\left|s^{n}-s^{q}\right|^{2}}+\frac{\delta c}{t^{1 / k} s^{\langle a ; s\rangle / k}} \frac{1}{\left(s^{n}-s^{q}\right)^{1 / k}}\right) .
$$

Now, for positive $p$, analyze the sum

$$
\begin{aligned}
\sum_{q=1}^{\ell-1} \sum_{n=q+1}^{\ell} \frac{1}{\left(s^{n}-s^{q}\right)^{1 / p}} & \leq \sum_{q=1}^{\ell-1} \sum_{n=q+1}^{\ell} \frac{1}{\left(s^{n} / 2\right)^{1 / p}} \\
& \leq 2^{1 / p} \sum_{q=1}^{\ell-1} \sum_{n=q+1}^{\ell} s^{-n / p} \\
& \leq 2^{1 / p} \sum_{q=1}^{\ell-1} \int_{q}^{\ell} s^{-x / p} d x \\
& \leq 2^{1 / p} \sum_{q=1}^{\ell-1} \frac{p}{\log (s)} s^{-q / p} \\
& \leq 2^{1 / p} \frac{p}{\log (s)} \int_{0}^{\ell} s^{-x / p} d x \\
& \leq 2^{1 / p}\left(\frac{p}{\log (s)}\right)^{2} .
\end{aligned}
$$

Hence,

$$
Z \leq \frac{1}{\ell}+\frac{2}{\ell^{2}} \frac{16}{t^{2}} 2^{2}\left(\frac{1 / 2}{\log (s)}\right)^{2}+\frac{2}{\ell^{2}} \frac{\delta c}{t^{1 / k} s^{\langle a ; s\rangle / k}} 2^{1 / k}\left(\frac{k}{\log (s)}\right)^{2} .
$$

By noting that $t$ is a positive integer and choosing $M$ so that for every $m \geq M, \delta$ is sufficiently small,

$$
Z \leq \frac{1}{\ell}+\frac{32}{\ell^{2} \log (s)^{2}}+\frac{1}{\ell^{2}}<\frac{100}{\ell} .
$$

By [3, page 314], $M$ is uniformly computable from the other parameters of the construction. 
Definition 9. Let $s$ be a base. Let $I$ be a dyadic interval of length $2^{-a}$. Let $\ell$ and $t$ be positive integers, and $\epsilon$ be a positive real. We define

$$
A(I, \ell, s, t, \epsilon)=\left\{x \in I:\left|\frac{1}{\ell} \sum_{n=\langle a ; s\rangle+1}^{\langle a ; s\rangle+\ell} e\left(2 \pi i t s^{n} x\right)\right| \geq \epsilon\right\} .
$$

The following is an adaptation of an argument due to Davenport, Erdös and LeVeque in [8] reproduced as Lemma 1.8 in [7].

Lemma 10. Let $s$ be a base, $I$ be a dyadic interval of length $2^{-a}$ and $k$ a positive integer. Let $t$ be a positive integer. Let $\epsilon$ and $\delta$ be positive reals with $\epsilon$ less than $1 / 2$. Then there are positive integers $M$ and $L$ such that for all $m$ greater than or equal to $M$,

$$
\nu_{I, m, k}\left(\bigcup_{\ell \geq L} A(I, \ell, s, t, \epsilon)\right)<\delta .
$$

Furthermore, $L$ is uniformly computable from $k, \epsilon, \delta, s$ and $t$, and does not depend upon $I$. On the other hand, $M$ is uniformly computable from $I, k, s$ and $t$.

Proof. By the definition of $A(I, \ell, s, t, \gamma)$,

$$
\gamma^{2} \nu_{I, m, k}(A(I, \ell, s, t, \gamma)) \leq \int \frac{1}{\ell^{2}}\left|\sum_{n=\langle a ; s\rangle+1}^{\langle a ; s\rangle+\ell} e\left(2 \pi i t s^{n} x\right)\right|^{2} d \nu_{I, m, k}(x) .
$$

By Lemma 8, there is an $M$ such that for all $m \geq M$ and all positive integers $\ell$,

$$
\int \frac{1}{\ell^{2}}\left|\sum_{n=\langle a ; s\rangle+1}^{\langle a ; s\rangle+\ell} e\left(2 \pi i t s^{n} x\right)\right|^{2} d \nu_{I, m, k}(x)<\frac{100}{\ell} .
$$

We fix this value of $M$ and let $m$ be greater than or equal to it. Then,

$$
\nu_{I, m, k}(A(I, \ell, s, t, \gamma))<\frac{100}{\gamma^{2} \ell} .
$$

Note that the above inequality holds for any real number $\gamma$. For the rest of the proof we fix parameters $I, s, t, \gamma=\epsilon / 7$ and abbreviate $A(I, \ell, s, t, \epsilon / 7)$ by writing $A_{\ell}$, for varying values of $\ell$. Set $\ell_{1}=1$ and $\ell_{j+1}=\left\lceil\ell_{j} /(1-\epsilon / 7)\right\rceil+1$ for $j \geq 1$ such that

$$
\frac{\ell_{j+1}-\ell_{j}}{\ell_{j+1}}>\frac{\epsilon}{7} \quad \text { and } \quad \frac{\ell_{j+1}-\ell_{j}}{\ell_{j}}<2 \frac{\epsilon}{7}+\frac{2}{\ell_{j}} \text {. }
$$

For each $j$, let $h_{j}$ be the integer in $\left[\ell_{j}, \ell_{j+1}\right)$ that minimizes $\nu_{I, m, k}\left(A_{h_{j}}\right) / h_{j}$. Hence,

$$
\sum_{\ell=\ell_{j}}^{\ell_{j+1}-1} \frac{\nu_{I, m, k}\left(A_{\ell}\right)}{\ell} \geq\left(\ell_{j+1}-\ell_{j}\right) \frac{\nu_{I, m, k}\left(A_{h_{j}}\right)}{h_{j}} \geq \frac{\epsilon}{7} \nu_{I, m, k}\left(A_{h_{j}}\right) .
$$

Thus,

$$
100 \frac{7^{3}}{\epsilon^{3}} \sum_{\ell=\ell_{j}}^{\ell_{j+1}-1} \frac{1}{\ell^{2}}>\nu_{I, m, k}\left(A_{h_{j}}\right)
$$

and so for every $j$,

$$
100 \frac{7^{3}}{\epsilon^{3}} \frac{1}{\ell_{j}-1}>\sum_{p=j}^{\infty} \nu_{I, m, k}\left(A_{h_{p}}\right) .
$$


Let $j_{0}$ be minimal such that

$$
\ell_{j}-1 \geq \frac{100}{\delta} \frac{7^{3}}{\epsilon^{3}} \text { and } \quad \frac{2}{\ell_{j_{0}}}<\frac{\epsilon}{7}
$$

Note that $j_{0}$ does not depend on $I$. Then, by the first inequality,

$$
\delta>\sum_{j=j_{0}}^{\infty} \nu_{I, m, k}\left(A_{h_{j}}\right) .
$$

Now, consider an $x \in I$ such that for all $j \geq j_{0}, x \notin A_{h_{j}}$, or equivalently,

$$
\left|\frac{1}{h_{j}} \sum_{\ell=\langle a ; s\rangle+1}^{\langle a ; s\rangle+h_{j}} e\left(2 \pi i t s^{\ell} x\right)\right|<\frac{\epsilon}{7},
$$

and let $\ell$ be a positive integer greater than or equal to $\ell_{j_{0}}$. Let $j$ be such that $\ell_{j} \leq \ell<\ell_{j+1}$. Note that,

$$
\begin{gathered}
\frac{1}{\ell} \sum_{n=\langle a ; s\rangle+1}^{\langle a ; s\rangle+\ell} e\left(2 \pi i t s^{n} x\right)-\frac{1}{h_{j}} \sum_{n=\langle a ; s\rangle+1}^{\langle a ; s\rangle+h_{j}} e\left(2 \pi i t s^{n} x\right) \\
=\frac{1}{\ell}\left(\sum_{n=\langle a ; s\rangle+1}^{\langle a ; s\rangle+\ell} e\left(2 \pi i t s^{n} x\right)-\sum_{n=\langle a ; s\rangle+1}^{\langle a ; s\rangle+h_{j}} e\left(2 \pi i t s^{n} x\right)\right) \\
+\left(\frac{1}{\ell}-\frac{1}{h_{j}}\right) \sum_{n=\langle a ; s\rangle+1}^{\langle a ; s\rangle+h_{j}} e\left(2 \pi i t s^{n} x\right) .
\end{gathered}
$$

It follows that for such $x$,

$$
\begin{aligned}
& \left|\frac{1}{\ell} \sum_{n=\langle a ; s\rangle+1}^{\langle a ; s\rangle+\ell} e\left(2 \pi i t s^{n} x\right)-\frac{1}{h_{j}} \sum_{n=\langle a ; s\rangle+1}^{\langle a ; s\rangle+h_{j}} e\left(2 \pi i t s^{n} x\right)\right| \\
& <\left(\frac{\ell_{j+1}-\ell_{j}}{\ell_{j}}\right)+\left(\frac{\ell_{j+1}-\ell_{j}}{\ell_{j}}\right) \\
& <\left(2 \frac{\epsilon}{7}+\frac{2}{\ell_{j}}\right)+\left(2 \frac{\epsilon}{7}+\frac{2}{\ell_{j}}\right) \\
& <6 \frac{\epsilon}{7}
\end{aligned}
$$

and by the triangle inequality, for this $x$,

$$
\left|\frac{1}{\ell} \sum_{\ell=\langle a ; s\rangle+1}^{\langle a ; s\rangle+\ell} e\left(2 \pi i t s^{\ell} x\right)\right|<\left|\frac{1}{h_{j}} \sum_{\ell=\langle a ; s\rangle+1}^{\langle a ; s\rangle+h_{j}} e\left(2 \pi i t s^{\ell} x\right)\right|+6 \frac{\epsilon}{7}<\frac{\epsilon}{7}+6 \frac{\epsilon}{7}=\epsilon .
$$

Then, $x \notin A(I, \ell, s, t, \epsilon)$. So, $\bigcup_{\ell \geq \ell_{j}} A(I, \ell, s, t, \epsilon)$ is contained in $\bigcup_{j \geq j_{0}} A_{h_{j}}$ and thereby $\nu_{I, m, k}\left(\bigcup_{\ell \geq \ell_{j_{0}}} A(I, \ell, s, t, \epsilon)\right)<\delta$. This proves the lemma for $M$ as above and $L$ is equal to $\ell_{j_{0}}$, with the observation that since $j_{0}$ did not depend on $I$ neither does $L$.

Lemma 11. Let $S$ be a set of bases, $I$ be a dyadic interval of length $2^{-a}$ and $k$ a positive integer. Let $\epsilon$ be a positive real number. Then, there are positive integers $M$ and $L$ such that for every $m \geq M$, 


$$
\begin{aligned}
& \nu_{I, m, k}(I)=1, \text { and } \\
& \nu_{I, m, k}\left(\left\{y \in I: \forall s \in S, \forall \ell \geq L, D\left(\left\{s^{j} y\right\}:\langle a ; s\rangle \leq j<\langle a+\ell ; s\rangle\right)<\epsilon\right\}\right) \geq \frac{1}{2} .
\end{aligned}
$$

Furthermore, $L$ is uniformly computable from $k, S$ and $\epsilon$, and does not depend on $I$; $M$ is uniformly computable from $I, k, S$ and $\epsilon$.

Proof. Let $T$ and $\gamma$ be, respectively, the set of positive integers and the real number determined by Lemma 7 with input $\epsilon$.

For each $s \in S$ and $t \in T$ apply Lemma 10 with $\epsilon$ equal to $\gamma$, and with $\delta$ equal to $1 /(2 \# T \# S)$. Let $M_{0}$ and $L_{0}$ be, respectively, the maximum of the values of $M$ and $L$ output by Lemma 10 in each case. Then, for every $m \geq M_{0}$,

$$
\sum_{t \in T} \sum_{s \in S} \nu_{I, m, k}\left(\bigcup_{\ell \geq L_{0}} A(I, \ell, s, t, \gamma)\right)<\sum_{t \in T} \sum_{s \in S} \frac{1}{2 \# T \# S}=\frac{1}{2} .
$$

The proof is completed by taking $L$ to be the minimum integer such that for every $s \in S,\langle a+L ; s\rangle \geq\langle a ; s\rangle+L_{0}$, and by taking $M$ to be the maximum between $M_{0}$ and the value $M$ determined by Lemma 6 .

Lemma 12. For every base $s$ and for every positive real $\epsilon$ there is an integer $L$ with the following property. Let $[u, v]$ be any subinterval of $[0,1]$ such that

$$
2 \epsilon<v-u, \quad 2 \epsilon<u \text { in case } u \neq 0, \text { and } 2 \epsilon<1-v \text { in case } v \neq 1 \text {. }
$$

For all $\ell \geq L$ and for all real numbers $x$ and $y$ satisfying $|x-y|<2^{-\ell}$, if for each interval $J \subseteq[0,1]$ with endpoints in $\{0, u \pm \epsilon, v \pm \epsilon, 1\}, D\left(J,\left(\left\{s^{j} x\right\}: j<\langle\ell ; s\rangle\right)\right)<\epsilon$, then

$$
D\left([u, v],\left(\left\{s^{j} y\right\}: j<\langle\ell ; s\rangle\right)\right)<8 \epsilon .
$$

Proof. Let $s,[u, v]$ and $\epsilon$ be fixed as above. Let $L$ be an integer such that $\left\lceil\left|\log _{s}(\epsilon)\right|\right\rceil$ is less than $\epsilon\langle L ; s\rangle$. Suppose that $\ell$ is greater than or equal to $L$ and that $x$ and $y$ are real numbers such that $|x-y|<1 / 2^{\ell}$ and for each interval $J$ with endpoints contained in $\{0, u \pm \epsilon, v \pm \epsilon, 1\} D\left(J,\left(\left\{s^{j} x\right\}: j<\langle\ell ; s\rangle\right)\right)<\epsilon$.

We first show that $\#\left\{j:\left\{s^{j} y\right\} \in[u, v]\right.$ and $\left.0 \leq j<\langle\ell ; s\rangle\right\}$ is greater than $(|v-u|-8 \epsilon)\langle\ell ; s\rangle$. Consider the interval $[u+\epsilon, v-\epsilon]$. By assumption,

$$
\begin{aligned}
& \#\left\{j:\left\{s^{j} x\right\} \in[u+\epsilon, v-\epsilon] \text { and } 0 \leq j<\langle\ell ; s\rangle\right\} \\
& >(((v-\epsilon)-(u+\epsilon))-\epsilon)\langle\ell ; s\rangle \\
& >((v-u)-3 \epsilon)\langle\ell ; s\rangle .
\end{aligned}
$$

If $s^{j} 2^{-\ell}$ is less than $\epsilon$ and $x \in[u+\epsilon, v-\epsilon]$, then $y \in[u, v]$. Further, $s^{j} 2^{-\ell}>\epsilon$ only when $j>\langle\ell ; s\rangle-\left|\log _{s}(\epsilon)\right|$ and, by choice of $L$, there are at most $\epsilon\langle\ell ; s\rangle$ many such integers $j$ less than or equal to $\langle\ell ; s\rangle$. Then,

$$
\begin{aligned}
\#\left\{j:\left\{s^{j} y\right\} \in[u, v] \text { and } 0 \leq j<\langle\ell ; s\rangle\right\} & >((v-u)-3 \epsilon)\langle\ell ; s\rangle-\epsilon\langle\ell ; s\rangle \\
& >((v-u)-4 \epsilon)\langle\ell ; s\rangle .
\end{aligned}
$$

Similarly, regarding only the fully nontrivial case in which $0<u$ and $v<1$,

$$
\begin{aligned}
& \#\left\{j:\left\{s^{j} y\right\} \in[0, u] \text { and } 0 \leq j<\langle\ell ; s\rangle\right\}>(u-4 \epsilon)\langle\ell ; s\rangle, \\
& \#\left\{j:\left\{s^{j} y\right\} \in[v, 1] \text { and } 0 \leq j<\langle\ell ; s\rangle\right\}>((1-v)-4 \epsilon)\langle\ell ; s\rangle,
\end{aligned}
$$


and so

$$
\begin{aligned}
& ((v-u)+8 \epsilon)\langle\ell ; s\rangle \\
& >\#\left\{j:\left\{s^{j} y\right\} \in[u, v] \text { and } 0 \leq j<\langle\ell ; s\rangle\right\} \\
& >((v-u)-8 \epsilon)\langle\ell ; s\rangle
\end{aligned}
$$

as required.

Lemma 13. Let $\epsilon$ be a positive real. Let any sequence of reals in the unit interval $\left(x_{1}, \ldots, x_{\ell}\right)$ of length $\ell$ be such that $D\left(x_{1}, \ldots, x_{\ell}\right)<\epsilon$. Let $\left(y_{1}, \ldots, y_{n}\right)$ be any sequence of reals in the unit interval, of length $n$ such that $n<\epsilon \ell$. Then for all $k \leq n, D\left(x_{1}, \ldots, x_{\ell}, y_{1}, \ldots, y_{k}\right)<2 \epsilon$ and $D\left(y_{1}, \ldots, y_{k}, x_{1} \ldots, x_{\ell}\right)<2 \epsilon$.

Proof. Immediate from the definition.

\section{Proof of the Theorem}

For $n$ an integer greater than or equal to 2 and $\epsilon$ a positive real number, let $L_{11}(n, \epsilon)$ and $L_{12}(n, \epsilon)$ be the supremum of the output numbers $L$ in Lemmas 11 and 12, respectively, for inputs $s$, a base less than or equal to $n$, Liouville exponent $k$ equal to $n$, and $\epsilon$, a positive real number. Without loss of generality, we assume that $L_{11}$ and $L_{12}$ increase as the first argument increases and as the second argument decreases.

Definition 14. An interval $I \subseteq[0,1]$ meets the Liouville condition for exponent $k$ if for any real $x \in I$ there is an integer $q>1$ such that $\|q x\|<q^{-k}$.

Then, a real number is Liouville when for each exponent $k$ there is an interval that contains $x$ and meets the Liouville condition for exponent $k$.

4.1. Algorithm. We proceed by recursion to define a sequence of dyadic intervals $\left[x_{n}, x_{n}+2^{-a_{n}}\right)$, that is to say that $a_{n}$ is a nonnegative integer and $x_{n}$ is of the form $p / 2^{a_{n}}$ with $0 \leq p<2^{a_{n}}$. To simplify notation, let $\epsilon_{n}=1 / 8^{n}$.

Let $x_{0}=0$ and $a_{0}=0$. Given $\left[x_{n}, x_{n}+2^{-a_{n}}\right.$ ) from the previous step, let $\left[x_{n+1}, x_{n+1}+2^{-a_{n+1}}\right)$ be the dyadic interval minimizing $a_{n+1}$ and breaking ties by minimizing $x_{n}$, with the following conditions:

- $\left[x_{n+1}, x_{n+1}+2^{-a_{n+1}}\right) \subseteq\left[x_{n}, x_{n}+2^{-a_{n}}\right)$.

- $\left[x_{n+1}, x_{n+1}+2^{-a_{n+1}}\right]$ meets the Liouville condition for exponent $n+1$.

- $a_{n+1}>L_{12}\left(n+1, \epsilon_{n+1} / 16\right)$.

- For every base $s$ less than or equal to $n+1$,

$$
\left\langle a_{n+1} ; s\right\rangle \epsilon_{n+1} / 16>\left\langle L_{11}\left(n+2, \epsilon_{n+2} / 16\right) ; s\right\rangle .
$$

- For every base $s$ less than or equal to $n$, for all nontrivial intervals $J \subseteq[0,1]$ with rational endpoints of the form $\left[p_{1} / 8^{n-2}, p_{2} / 8^{n-2}\right]$ and for all integers $\ell \in\left[a_{n}, a_{n+1}\right)$,

$$
D\left(J,\left(\left\{s^{j} x_{n+1}\right\}: j<\langle\ell ; s\rangle\right)\right)<128 \epsilon_{n} .
$$

- For every base $s$ less than or equal to $n+1$, for all nontrivial intervals $J \subseteq[0,1]$ with rational endpoints of the form $\left[p_{1} / 8^{n+1}, p_{2} / 8^{n+1}\right]$,

$$
D\left(J,\left(\left\{s^{j} x_{n+1}\right\}: j<\left\langle a_{n+1} ; s\right\rangle\right)\right)<\epsilon_{n+1} .
$$


4.2. Verification. We first check by induction that the sequence $\left(x_{n}: n \geq 0\right)$ is well defined. We have specified $x_{0}$ and $a_{0}$ explicitly. It is immediate that for step $n=1$ there is a suitable choice for $x_{1}$ and $a_{1}$. Assume that the sequence is defined up to and including $\left[x_{n}, x_{n}+2^{-a_{n}}\right)$, where $n \geq 1$. Let $I$ be the interval $\left[x_{n}, x_{n}+2^{-a_{n}}\right]$ and let $S$ be the set of bases less than or equal to $n+1$. Apply Lemma 11 for $\epsilon=\epsilon_{n+1} / 16, k=n+1, I, a=a_{n}$ and $S$. Obtaining $L_{11}\left(n+1, \epsilon_{n+1} / 16\right)=L$ and $m=M$.

Let $a$ be a positive integer with the following properties:

- $a>(n+3) \log _{2} m$.

- $a>L_{12}\left(n+1, \epsilon_{n+1} / 16\right)$.

- For every base $s$ less than or equal to $n+1$,

$$
\langle a ; s\rangle \epsilon_{n+1} / 16>\left\langle L_{11}\left(n+2, \epsilon_{n+2} / 16\right) ; s\right\rangle .
$$

- For every base $s$ less than or equal to $n+1$,

$$
\left(\langle a ; s\rangle-\left\langle a_{n} ; s\right\rangle\right) \epsilon_{n+1} / 16>\left\langle a_{n} ; s\right\rangle .
$$

Let $Y$ be the set of reals $y \in I$ such that $\forall s \in S, \forall \ell \geq L_{11}\left(n+1, \epsilon_{n+1} / 16\right)$,

$$
D\left(\left\{s^{j} y\right\}:\left\langle a_{n} ; s\right\rangle \leq j<\left\langle a_{n}+\ell ; s\right\rangle\right)<\epsilon_{n+1} / 16 .
$$

By definition, $Y$ satisfies

$$
\nu_{I, m, n+1}(Y) \geq \frac{1}{2} .
$$

Fix a real number $y \in Y$, which implies $y \in E(m, n+1) \cap I$ and for every $s \in S$ and every $\ell \geq L_{11}\left(n+1, \epsilon_{n+1} / 16\right)$,

$$
D\left(\left\{s^{j} y\right\}:\left\langle a_{n} ; s\right\rangle \leq j<\left\langle a_{n}+\ell ; s\right\rangle\right)<\epsilon_{n+1} / 16 .
$$

By inductive hypothesis, $a_{n}>L_{12}\left(n, \epsilon_{n} / 16\right) \geq L_{12}\left(n, \epsilon_{n}\right)$ and for every base $s \leq n$ and all nontrivial intervals $J \subseteq[0,1]$ with rational endpoints of the form $\left[p_{1} / 8^{n}, p_{2} / 8^{n}\right]$

$$
D\left(J,\left(\left\{s^{j} x_{n}\right\}: j<\left\langle a_{n} ; s\right\rangle\right)\right)<\epsilon_{n} .
$$

Let $J^{*}$ be a subinterval of $[0,1]$ of the form $\left[p_{1} / 8^{n-1}, p_{2} / 8^{n-1}\right]$. Then, Lemma 12 applies to the pair $x_{n}$ and $y$ and the interval $J^{*}$ to conclude that

$$
D\left(J^{*},\left(\left\{s^{j} y\right\}: j<\left\langle a_{n} ; s\right\rangle\right)\right)<8 \epsilon_{n} .
$$

By inductive hypothesis again, for all $s \leq n$, we have

$$
\left\langle a_{n} ; s\right\rangle \epsilon_{n} / 16>\left\langle L_{11}\left(n+1, \epsilon_{n+1} / 16\right) ; s\right\rangle,
$$

and so by Lemma 13, for all $\ell \leq L_{11}\left(n+1, \epsilon_{n+1} / 16\right)$ and all $s \leq n$,

$$
D\left(J^{*},\left(\left\{s^{j} y\right\}: j<\left\langle a_{n}+\ell ; s\right\rangle\right)\right)<16 \epsilon_{n} .
$$

If $\ell$ is such that $L_{11}\left(n+1, \epsilon_{n+1} / 16\right)<\ell \leq a-a_{n}$, then $\left(\left\{s^{j} y\right\}: j<\left\langle a_{n}+\ell ; s\right\rangle\right)$ is the concatenation of $\left(\left\{s^{j} y\right\}: j<\left\langle a_{n} ; s\right\rangle\right)$ and $\left(\left\{s^{j} y\right\}:\left\langle a_{n} ; s\right\rangle \leq j<\left\langle a_{n}+\ell ; s\right\rangle\right)$, both of which have discrepancy, with respect to interval $J^{*}$, less than $8 \epsilon_{n}$, and so it has discrepancy less than $8 \epsilon_{n}$. Thus, for every $\ell$ between $a_{n}$ and $a$,

$$
D\left(J^{*},\left(\left\{s^{j} y\right\}: j<\langle\ell ; s\rangle\right)\right)<16 \epsilon_{n} .
$$

Let $\left[\bar{y}, \bar{y}+2^{-a}\right)$ be a dyadic interval such that $y-\bar{y}<2^{-a}$. As above for $x_{n}$ and $y$, Lemma 12 applies to the pair $y$ and $\bar{y}$ to conclude that for every $\ell$ between $a_{n}$ and $a$ and every subinterval $J$ of $[0,1]$ of the form $\left[p_{1} / 8^{n-2}, p_{2} / 8^{n-2}\right]$,

$$
D\left(J,\left(\left\{s^{j} \bar{y}\right\}: j<\langle\ell ; s\rangle\right)\right)<8 \cdot 16 \epsilon_{n}=128 \epsilon_{n} .
$$


Further, for every $s \leq n+1$, since $D\left(\left\{s^{j} y\right\}:\left\langle a_{n} ; s\right\rangle \leq j<\langle a ; s\rangle\right) \leq \epsilon_{n+1} / 16$ (obtained above) and $\left(\langle a ; s\rangle-\left\langle a_{n} ; s\right\rangle\right) \epsilon_{n+1} / 16 \geq\left\langle a_{n} ; s\right\rangle$, for any interval $J \subseteq[0,1]$,

$$
D\left(J,\left(\left\{s^{j} y\right\}: j<\langle a ; s\rangle\right)\right)<2 \cdot \epsilon_{n+1} / 16=\epsilon_{n+1} / 8,
$$

and so, by Lemma 13

$$
D\left(J,\left(\left\{s^{j} \bar{y}\right\}: j<\langle a ; s\rangle\right)\right)<8 \cdot \epsilon_{n+1} / 8=\epsilon_{n+1} .
$$

Finally, since $y \in E(m, n+1)$, let $q$ be a positive prime such that $m \leq q$ and $\|q y\|<q^{-n-2}$. Let $y^{*} \in\left[\bar{y}, \bar{y}+2^{-a}\right)$. We verify that $\left\|q y^{*}\right\|<q^{-n-1}$.

Since $y-\bar{y}<2^{-a}$, then, $y-y^{*}<2^{-a}$.

Since $a>(n+3) \log _{2} m$, then, $2^{-a}<m^{-n-3}<q^{-n-3}(q-1)$.

Thus,

$$
\begin{aligned}
q 2^{-a} & <q^{-n-2}(q-1), \\
q^{-n-2}+q 2^{-a} & <q^{-n-1}, \\
|| q y \|+\left|q y-q y^{*}\right| & <q^{-n-1}, \\
|| q y^{*} \| & <q^{-n-1}, \text { as required. }
\end{aligned}
$$

Hence, $\left[\bar{y}, \bar{y}+2^{-a}\right)$ satisfies all requirements to be a dyadic interval for step $n+1$.

Now, let $x$ be the limit of the sequence $\left(x_{n}: n \geq 0\right)$. By virtue of the second condition in the specification of $x_{n+1}$ from $x_{n}, x$ is a Liouville number. To check that $x$ is absolutely normal, let $s$ be a base, $\epsilon$ a real number and $J$ an interval. By a continuity argument, we may fix positive integer $m$ and assume that the endpoints of $J$ are dyadic rational numbers of the form $p / 8^{m}$. Consider $N$ so large that $s \leq N$, $m<N-3$ and $\epsilon>8 \epsilon_{N}$. Let $\ell$ be a positive integer greater than or equal to $a_{N}$. Let $n$ be such that $a_{n} \leq \ell<a_{n+1}$. By the choice of $x_{n+1}$, Lemma 12 applies to $x_{n+1}$ and $x$ to conclude that

$$
D\left(J,\left(\left\{s^{j} x\right\}: j<\langle\ell ; s\rangle\right)\right)<8 \cdot 128 \epsilon_{n} .
$$

Since the sequence $\epsilon_{n}$ goes to zero as $n$ goes to infinity, the discrepancy of

$$
\left(\left\{s^{j} x\right\}: j<\langle\ell ; s\rangle\right)
$$

goes to zero as $\ell$ goes to infinity. Hence, $x$ is absolutely normal. This completes the proof.

\section{ACKNOWLEDGEMENTS}

The authors thank Yann Bugeaud for bringing the question in this paper to our attention and Michael Christ for early advice on harmonic analysis. Becher and Heiber are members of Laboratoire International Associé INFINIS, CONICET/Universidad de Buenos Aires - CNRS/Université Paris Diderot.

\section{REFERENCES}

[1] V. Becher, P. A. Heiber, and T. A. Slaman, A polynomial-time algorithm for computing absolutely normal numbers, Inform. and Comput. 232 (2013), 1-9, DOI 10.1016/j.ic.2013.08.013. MR.3132518

[2] V. Becher and T. A. Slaman, On the normality of numbers to different bases, preprint, arXiv:1311.0333, 2013.

[3] C. Bluhm, On a theorem of Kaufman: Cantor-type construction of linear fractal Salem sets, Ark. Mat. 36 (1998), no. 2, 307-316, DOI 10.1007/BF02384771. MR.1650442 (99i:43009) 
[4] C. E. Bluhm, Liouville numbers, Rajchman measures, and small Cantor sets, Proc. Amer. Math. Soc. 128 (2000), no. 9, 2637-2640, DOI 10.1090/S0002-9939-00-05276-X. MR.1657762 (2000m:11063)

[5] É. Borel, Les probabilités dénombrables et leurs applications arithmétiques, Supplemento di Rendiconti del circolo matematico di Palermo, 27 1909, 247-271

[6] Y. Bugeaud, Nombres de Liouville et nombres normaux (French, with English and French summaries), C. R. Math. Acad. Sci. Paris 335 (2002), no. 2, 117-120, DOI 10.1016/S1631073X(02)02456-1. MR1920005 (2003e:11081)

[7] Y. Bugeaud, Distribution Modulo One and Diophantine Approximation, Cambridge Tracts in Mathematics, vol. 193, Cambridge University Press, Cambridge, 2012. MR 2953186

[8] H. Davenport, P. Erdős, and W. J. LeVeque, On Weyl's criterion for uniform distribution, Michigan Math. J. 10 (1963), 311-314. MR0153656 (27 \#3618)

[9] L. Kuipers and H. Niederreiter, Uniform distribution of sequences, Dover, 2006.

[10] Satyadev Nandakumar and Santhosh Kumar Vangapelli, Normality and finite-state dimension of Liouville numbers, preprint, arXiv:1204.4104, 2012.

[11] H. Weyl, Über die Gleichverteilung von Zahlen mod. Eins (German), Math. Ann. 77 (1916), no. 3, 313-352, DOI 10.1007/BF01475864. MR1511862

Departmento de Computación, Facultad de Ciencias Exactas y Naturales, UniversiDAd De Buenos Aires \& CONICET, Argentina

E-mail address: vbecher@dc.uba.ar

Departmento de Computación, Facultad de Ciencias Exactas y Naturales, UniversiDAD De Buenos Aires \& CONICET, Argentina

E-mail address: pheiber@dc.uba.ar

The University of California, Berkeley, Department of Mathematics, 719 Evans Hall \#3840, Berkeley, California 94720-3840

E-mail address: slaman@math.berkeley.edu 